JOURNAL OF UNIVERSAL MATHEMATICS

VOL.1 No.3 PP.261-268 (2018)

ISSN-2618-5660

\title{
ON INTERVAL VALUED INTUITIONISTIC FUZZY PAIRS
}

\author{
KRASSIMIR ATANASSOV, PETER VASSILEV, EULALIA SZMIDT, AND JANUSZ KACPRZYK
}

In memory of our dear friend Beloslav Riečan (1936-2018)

\section{INTRODUCTION}

As was mentioned in [9], the first researches, related to Intuitionistic Fuzzy Sets (IFSs) started in 1983 and from the beginning, the concept of Intuitionistic Fuzzy Pair (IFP) had started to be used. As we mentioned in [9], a lot of our colleagues working in the area of the intuitionistic fuzziness, used it without a special definition in many of their publications, using different names: IFP, intuitionistic fuzzy couple, intuitionistic fuzzy value and others. In the mentioned paper, we proposed to the researchers in the area of the intuitionistic fuzziness to use only one name for the concept.

Here, we give a formal definition of an Interval Valued IFP (IVIFP) and definitions of operations, relations and operators, defined oved IVIFPs by analogy with the IFPs and of the IFSs and Interval Values IFSs (IVIFSs), published during the last already 35 years, using our books $[2,3,13]$.

\section{DEFINITION AND GEOMETRICAL INTERPRETATIONS OF AN IVIFP}

The Interval Valued Intuitionistic Fuzzy Pair (IVIFP) is an object with the form $\langle M, N\rangle$, where $M, N \subseteq[0,1]$ are closed sets, $M=[\inf M, \sup M], N=[\inf N, \sup N]$ and $\sup M+\sup N \leq 1$, that is used as an evaluation of some object or process and which components $(M$ and $N)$ are interpreted as intervals of degrees of membership and nonmembership, or intervals of degrees of validity and non-validity, or intervals of degree of correctness and non-correctness, etc. Two of the basic geometrical interpretations of an IVIFP are shown on Figs. 1 and 2.

For the needs of the discussion below, we define the notion of Intuitionistic Fuzzy Tautological Pair (IFTP) by:

$$
x \text { is an IFTP if and only if } \inf M \geq \sup N,
$$

while $x$ is a Tautological Pair (TP) iff $M=[1,1]$ and $N=[0,0]$.

Date: Accepted October 23, 2018. 


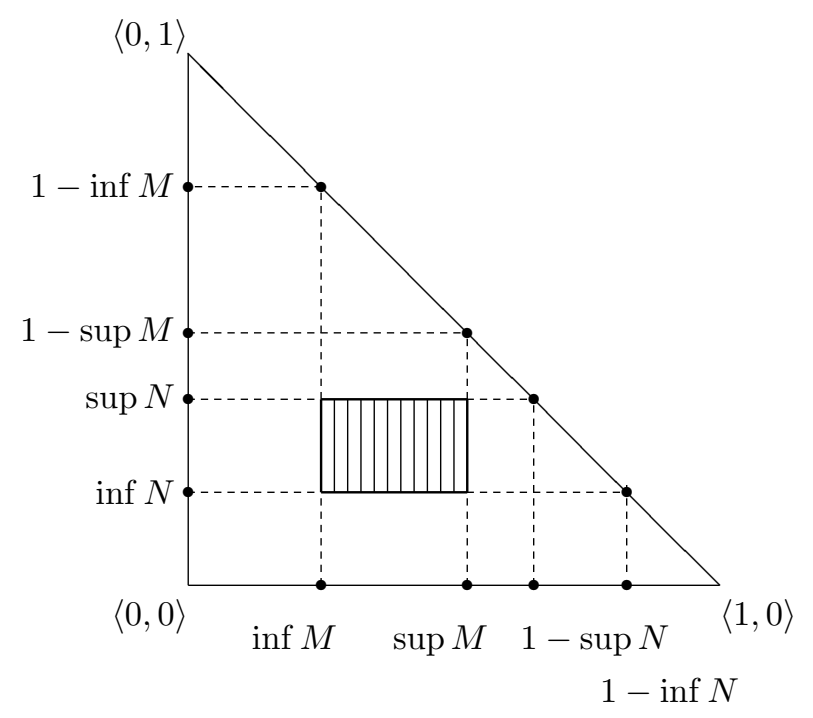

FIGURE 1. Geometrical interpretation of an IVIFP

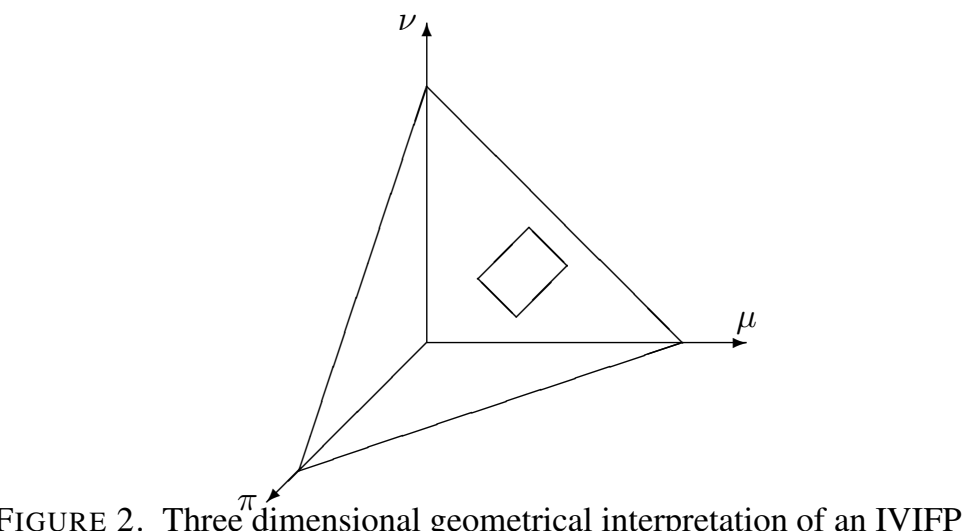

FIGURE 2. Three dimensional geometrical interpretation of an IVIFP

\section{RELATIONS OVER IVIFPS}

Let us have two IVIFPs $x=\langle M, N\rangle$ and $y=\langle P, Q\rangle$. We define the relations $x<y \operatorname{iff} \inf M<\inf P$ and $\sup M<\sup P$

$x<\diamond y$ iff $\inf N>\inf Q$ and $\sup N>\sup Q$

$x<y \operatorname{iff} \inf M<\inf P$ and $\sup M<\sup P$ and $\inf N>\inf Q$ and $\sup N>\sup Q$

$x \leq y \operatorname{iff} \inf M \leq \inf P$ and $\sup M \leq \sup P$

$x \leq \diamond y \operatorname{iff} \inf N \geq \inf Q$ and $\sup N \geq \sup Q$

$x \leq y$ iff $\inf M \leq \inf P$ and $\sup M \leq \sup P$ and $\inf N \geq \inf Q$ and $\sup N \geq \sup Q$

$x>y \operatorname{iff} \inf M>\inf P$ and $\sup M>\sup P$

$x>\diamond y \operatorname{iff} \inf N<\inf Q$ and $\sup N<\sup Q$

$x>y \operatorname{iff} \inf M>\inf P$ and $\sup M>\sup P$ and $\inf N<\inf Q$ and $\sup N<\sup Q$ 


$$
\begin{aligned}
& x \geq_{\square} y \operatorname{iff} \inf M \geq \inf P \text { and } \sup M \geq \sup P \\
& x \geq_{\diamond} y \text { iff } \inf N \leq \inf Q \text { and } \sup N \leq \sup Q \\
& x \geq y \operatorname{iff} \inf M \geq \inf P \text { and } \sup M \geq \sup P \text { and } \inf N \leq \inf Q \text { and } \sup N \leq \sup Q \\
& x=\square y i \inf M=\inf P \text { and } \sup M=\sup P \\
& x=\diamond y \operatorname{iff} \inf N=\inf Q \text { and } \sup N=\sup Q \\
& x=y \text { iff } \inf M=\inf P \text { and } \sup M=\sup P \text { and } \inf N=\inf Q \text { and } \sup N=\sup Q .
\end{aligned}
$$

\section{OPERATIONS OVER IVIFPS}

Now, there are 189 different intuitionistic fuzzy implications (since $\rightarrow_{40}$ and $\rightarrow_{173}$ coincide, see [14]) that generate 54 intuitionistic fuzzy negations and about 500 intuitionistic fuzzy conjunctions and the same number of intuitionistic fuzzy disjunctions. All of them have been introduced only for intuitionistic fuzzy case, but in a near future, they will be modified for interval valued intuitionistic fuzzy case, too. Here, we illustrate with two examples the process of this modification.

The first intuitionistic fuzzy implication (called first Zadeh's intuitionistic fuzzy implication) for IFP-case has the form

$$
x \rightarrow_{1} y=\langle\max (b, \min (a, c)), \min (a, d)\rangle,
$$

where (only here and in the next example) $x=\langle a, b\rangle, y=\langle c, d\rangle, a, b, c, d, a+b, c+d \in$ $[0,1]$, which in IVIFP-form is

$$
\begin{gathered}
x \rightarrow_{1} y=\langle[\max (\inf N, \min (\inf M, \inf P)), \max (\sup N, \min (\sup M, \sup P))], \\
[\min (\inf M, \inf Q), \min (\sup M, \sup Q)]\rangle .
\end{gathered}
$$

The second intuitionistic fuzzy implication (called Gödel's intuitionistic fuzzy implication) for IFP-case has the form

$$
x \rightarrow_{2} y=\langle 1-\operatorname{sg}(a-c), d \operatorname{sg}(a-c)\rangle,
$$

where for the real number $r$

$$
\operatorname{sg}(r)= \begin{cases}1 & \text { if } r>0 \\ 0 & \text { if } r \leq 0\end{cases}
$$

In IVIFP-form, the second Gödel's intuitionistic fuzzy implication is

$$
\begin{gathered}
x \rightarrow_{2} y=\langle[1-\operatorname{sg}(\inf M-\inf P), 1-\operatorname{sg}(\sup M-\sup P)], \\
[\inf Q \operatorname{sg}(\inf M-\inf P), \sup Q \operatorname{sg}(\sup M-\sup P)]\rangle .
\end{gathered}
$$

In the same manner we can construct the interval valued intuitionistic fuzzy conjunctions and disjunctions. For example, following [1], where the intuitionistic fuzzy conjunctions and disjunctions from the first type are described, we can construct the following 
operations:

$$
\begin{aligned}
x \&_{4} y=x \& y=\quad & \langle[\min (\inf M, \inf P), \min (\sup M, \sup N)], \\
& {[\max (\inf N, \inf Q), \max (\sup N, \sup Q)]\rangle } \\
x \vee_{4} y=x \vee y=\quad & \langle[\max (\inf M, \inf P), \max (\sup M \sup N)], \\
& {[\min (\inf N, \inf Q), \min (\sup N, \sup Q)]\rangle } \\
x \&_{2} y=x+y= & \langle[\inf M+\inf P-\inf M \inf P, \\
& \sup M+\sup P-\sup M \sup P], \\
& {[\inf N \inf Q, \sup N \sup Q]\rangle } \\
& \langle[\inf M \inf P, \sup M \sup P], \\
& {[\inf N+\inf Q-\inf N \inf Q,} \\
& \sup N+\sup Q-\sup N \sup Q]\rangle \\
& \left\langle\left[\frac{\inf M+\inf P}{2}, \frac{\sup M+\sup N}{2}\right],\left[\frac{\inf N+\inf Q}{2}, \frac{\sup N+\sup Q}{2}\right]\right\rangle .
\end{aligned}
$$

Analogously, we construct the interval valued intuitionistic fuzzy negations. For example, the first two implications generate the following two interval valued intuitionistic fuzzy negations:

$$
\begin{gathered}
\neg_{1} x=\langle N, M\rangle, \\
\neg_{2} x=\langle[1-\operatorname{sg}(\sup M), 1-\operatorname{sg}(\inf M)],[\operatorname{sg}(\inf M), \operatorname{sg}(\sup M)]\rangle .
\end{gathered}
$$

\section{OPERATORS OVER IVIFPS}

There are three types of modal operators over IFPs. The first of them is an intuitionistic fuzzy form of the standard modal operators (see, e.g., [12]).

Let as above, $x=\langle a, b\rangle$ be an IFP and let $\alpha, \beta \in[0,1]$. Then the modal type of operators defined over $x$ have the forms:

$$
\begin{array}{ll}
\square x= & \left\langle M_{A}(x),[\inf N, 1-\sup M]\right\rangle, \\
\nabla x= & \langle[\inf M, 1-\sup N], N\rangle, \\
D_{\alpha}(x)=\quad & \langle[\inf M, \sup M+\alpha(1-\sup M-\sup N)], \\
& {[\inf N, \sup N+(1-\alpha)(1-\sup M-\sup N]\rangle} \\
F_{\alpha, \beta}(x)=\quad & \langle[\inf M, \sup M+\alpha(1-\sup M-\sup N)], \\
& {[\inf N, \sup N+\beta(1-\sup M-\sup N)], \text { for } \alpha+\beta \leq 1,} \\
G_{\alpha, \beta}(x)= & \langle[\alpha \inf M, \alpha \sup M],[\beta \inf N, \beta \sup N]\rangle \\
H_{\alpha, \beta}(x)=\langle[\alpha \inf M, \alpha \sup M],[\inf N, \sup N+\beta(1-\sup M-\sup N)]\rangle, \\
H_{\alpha, \beta}^{*}(x)=\langle[\alpha \inf M, \alpha \sup M],[\inf N, \sup N+\beta(1-\alpha \sup M-\sup N)]\rangle, \\
J_{\alpha, \beta}(x)=\quad\langle[\inf M, \sup M+\alpha(1-\sup M-\sup N)],[\beta \inf N, \beta \sup N]\rangle, \\
J_{\alpha, \beta}^{*}(x)=\quad\langle[\inf M, \sup M+\alpha(1-\sup M-\beta \sup N)],[\beta \inf N, \beta \cdot \sup N]\rangle,
\end{array}
$$

where $\alpha, \beta \in[0,1]$. 


$$
\begin{aligned}
& \bar{F}\left(\begin{array}{ll}
\alpha & \gamma \\
\beta & \delta
\end{array}\right)(x)=\langle[\inf M+\alpha(1-\sup M-\sup N), \sup M+\beta(1-\sup M-\sup N)], \\
& [\inf N+\gamma(1-\sup M-\sup N), \sup N+\delta(1-\sup M-\sup N)]\rangle \text {, } \\
& \text { where } \beta+\delta \leq 1 \text {; } \\
& \bar{G}_{\left(\begin{array}{ll}
\alpha & \gamma \\
\beta & \delta
\end{array}\right)}(x)=\langle[\alpha \inf M, \beta \sup M],[\gamma \inf N, \delta \sup N]\rangle, \\
& \bar{H}\left(\begin{array}{ll}
\alpha & \gamma \\
\beta & \delta
\end{array}\right)(x)=\langle[\alpha \inf M, \beta \sup M], \\
& [\inf N+\gamma(1-\sup M-\sup N), \sup N+\delta(1-\sup M-\sup N)]\rangle \text {, } \\
& \bar{H}^{*}\left(\begin{array}{ll}
\alpha & \gamma \\
\beta & \delta
\end{array}\right)(x)=\langle[\alpha \inf M, \beta \sup M], \\
& [\inf N+\gamma(1-\beta \sup M-\sup N), \sup N+\delta(1-\beta \sup M-\sup N)]\rangle, \\
& \bar{J}\left(\begin{array}{ll}
\alpha & \gamma \\
\beta & \delta
\end{array}\right)(x)=\langle[\inf M+\alpha(1-\sup M-\sup N), \sup M+\beta(1-\sup M-\sup N)], \\
& [\gamma \inf N, \delta \sup N]\rangle, \\
& \bar{J}^{*}\left(\begin{array}{ll}
\alpha & \gamma \\
\beta & \delta
\end{array}\right)(x)=\langle[\inf M+\alpha(1-\delta \sup M-\sup N), \sup M+\beta(1-\sup M-\delta \sup N)] \text {, } \\
& [\gamma \inf N, \delta \sup N]\rangle,
\end{aligned}
$$

where $\alpha, \beta, \gamma, \delta \in[0,1]$ such that $\alpha \leq \beta$ and $\gamma \leq \delta$.

All these operators are partial cases of the following operator

$$
X\left(\begin{array}{llllll}
a_{1} & b_{1} & c_{1} & d_{1} & e_{1} & f_{1} \\
a_{2} & b_{2} & c_{2} & d_{2} & e_{2} & f_{2}
\end{array}\right)(x)
$$

$$
\begin{aligned}
= & \left\langle x,\left[a_{1} \inf M+b_{1}\left(1-\inf M-c_{1} \inf N\right), a_{2} \sup M+b_{2}\left(1-\sup M-c_{2} \sup N\right)\right],\right. \\
& {\left.\left[d_{1} \inf N+e_{1}\left(1-f_{1} \inf M-\inf N\right), d_{2} \sup N+e_{2}\left(1-f_{2} \sup M-\sup N\right)\right]\right\rangle, }
\end{aligned}
$$

where $a_{1}, b_{1}, c_{1}, d_{1}, e_{1}, f_{1}, a_{2}, b_{2}, c_{2}, d_{2}, e_{2}, f_{2} \in[0,1]$, the following four conditions are valid for $i=1,2$ :

$$
\begin{gathered}
a_{i}+e_{i}-e_{i} f_{i} \leq 1, \\
b_{i}+d_{i}-b_{i} \cdot c_{i} \leq 1, \\
b_{i}+e_{i} \leq 1, \\
a_{1} \leq a_{2}, b_{1} \leq b_{2}, c_{1} \leq c_{2}, d_{1} \leq d_{2}, e_{1} \leq e_{2}, f_{1} \leq f_{2} .
\end{gathered}
$$

The second type of operators is from another type (similar to modal one). Let $\alpha_{i}, \beta_{i}, \gamma_{i}, \delta_{i}, \varepsilon_{i}, \zeta_{i} \in$ $[0,1]$ for $i=1,2$. Then, we define

$$
\begin{aligned}
& \boxplus A=\left\langle\left[\frac{\inf M}{2}, \frac{\sup M}{2}\right],\left[\frac{\inf N_{A}(x)+1}{2}, \frac{\sup N_{A}(x)+1}{2}\right]\right\rangle, \\
& \boxplus_{A}=\left\langle\left[\frac{\inf M_{A}(x)+1}{2}, \frac{\sup M_{A}(x)+1}{2}\right],\left[\frac{\inf N}{2}, \frac{\sup N}{2}\right]\right\rangle,
\end{aligned}
$$




$$
\begin{aligned}
& \left.\Psi_{\left(\begin{array}{c}
\alpha_{1} \\
\alpha_{2}
\end{array}\right)}\right)^{A}=\left\langle\left[\alpha_{1} \inf M, \alpha_{2} \sup M\right]\right. \\
& \left.\left[\alpha_{1} \inf N_{A}(x)+1-\alpha_{1}, \alpha_{2} \sup N_{A}(x)+1-\alpha_{2}\right]\right\rangle, \\
& 凶\left(\begin{array}{c}
\alpha_{1} \\
\alpha_{2}
\end{array}\right) A=\left\langle\left[\alpha_{1} \inf M_{A}(x)+1-\alpha_{1}, \alpha_{2} \sup M_{A}(x)+1-\alpha_{2}\right]\right. \text {, } \\
& \left.\left[\alpha_{1} \inf N, \alpha_{2} \sup N\right]\right\rangle \\
& \boxplus\left(\begin{array}{ll}
\alpha_{1} & \beta_{1} \\
\alpha_{2} & \beta_{2}
\end{array}\right)^{A}=\left\langle\left[\alpha_{1} \inf M, \alpha_{2} \sup M\right]\right. \\
& \left.\left[\alpha_{1} \inf N_{A}(x)+\beta_{1}, \alpha_{2} \sup N_{A}(x)+\beta_{2}\right]\right\rangle, \\
& 凶\left(\begin{array}{ll}
\alpha_{1} & \beta_{1} \\
\alpha_{2} & \beta_{2}
\end{array}\right) A=\left\langle\left[\alpha_{1} \inf M_{A}(x)+\beta_{1}, \alpha_{2} \sup M_{A}(x)+\beta_{2}\right],\right. \\
& \left.\left[\alpha_{1} \inf N, \alpha_{2} \sup N\right]\right\rangle
\end{aligned}
$$

where $\alpha_{2}+\beta_{2} \leq 1$ and $\alpha_{1} \leq \alpha_{2}, \beta_{1} \leq \beta_{2}$,

$$
\begin{aligned}
& \boxplus\left(\begin{array}{lll}
\alpha_{1} & \beta_{1} & \gamma_{1} \\
\alpha_{2} & \beta_{2} & \gamma_{2}
\end{array}\right)^{A}=\left\langle\left[\alpha_{1} \inf M, \alpha_{2} \sup M\right],\right. \\
& \left.\left[\beta_{1} \inf N_{A}(x)+\gamma_{1}, \beta_{2} \sup N_{A}(x)+\gamma_{2}\right]\right\rangle, \\
& 凶\left(\begin{array}{ccc}
\alpha_{1} & \beta_{1} & \gamma_{1} \\
\alpha_{2} & \beta_{2} & \gamma_{2}
\end{array}\right) A=\left\langle\left[\alpha_{1} \inf M_{A}(x)+\gamma_{1}, \alpha_{2} \sup M_{A}(x)+\gamma_{2}\right]\right. \text {, } \\
& \left.\left[\beta_{1} \inf N, \beta_{2} \sup N\right]\right\rangle,
\end{aligned}
$$

where $\max \left(\alpha_{i}, \beta_{i}\right)+\gamma_{i} \leq 1$ for $i=1,2$ and $\alpha_{1} \leq \alpha_{2}, \beta_{1} \leq \beta_{2}, \gamma_{1} \leq \gamma_{2}$,

$$
\begin{aligned}
\bullet\left(\begin{array}{cccc}
\alpha_{1} & \beta_{1} & \gamma_{1} & \delta_{1} \\
\alpha_{2} & \beta_{2} & \gamma_{1} & \delta_{2}
\end{array}\right) A= & \left\langle\left[\alpha_{1} \inf M+\gamma_{1}, \alpha_{2} \sup M+\gamma_{2}\right],\right. \\
& {\left.\left[\beta_{1} \inf N_{A}(x)+\delta_{1}, \beta_{2} \sup N_{A}(x)+\delta_{2}\right]\right\rangle, }
\end{aligned}
$$

where $\max \left(\alpha_{i}, \beta_{i}\right)+\gamma_{i}+\delta_{i} \leq 1$ for $i=1,2$ and $\alpha_{1} \leq \alpha_{2}, \beta_{1} \leq \beta_{2}, \gamma_{1} \leq \gamma_{2}, \delta_{1} \leq \delta_{2}$,

$$
\begin{aligned}
& 0\left(\begin{array}{llllll}
\alpha_{1} & \beta_{1} & \gamma_{1} & \delta_{1} & \varepsilon_{1} & \zeta_{1} \\
\alpha_{2} & \beta_{2} & \gamma_{1} & \delta_{2} & \varepsilon_{1} & \zeta_{1}
\end{array}\right)^{A} \\
& =\left\langle\left[\alpha_{1} \inf M-\varepsilon_{1} \inf N_{A}(x)+\gamma_{1}, \alpha_{2} \sup M-\varepsilon_{2} \inf N_{A}(x)+\gamma_{2}\right],\right. \\
& \left.\left[\beta_{1} \inf N_{A}(x)-\zeta_{1} \inf M_{A}(x)+\delta_{1}, \beta_{2} \sup N_{A}(x)-\zeta_{2} \inf M_{A}(x)+\delta_{2}\right]\right\rangle,
\end{aligned}
$$

where $\alpha_{1} \leq \alpha_{2}, \beta_{1} \leq \beta_{2}, \gamma_{1} \leq \gamma_{2}, \delta_{1} \leq \delta_{2}, \varepsilon_{1} \geq \varepsilon_{2}, \zeta_{1} \leq \zeta_{2}$, and for $i=1,2$ :

$$
\begin{aligned}
& \max \left(\alpha_{i}-\zeta_{i}, \beta_{i}-\varepsilon_{i}\right)+\gamma_{i}+\delta_{i} \leq 1, \\
& \min \left(\alpha_{i}-\zeta_{i}, \beta_{i}-\varepsilon_{i}\right)+\gamma_{i}+\delta_{i} \geq 0 .
\end{aligned}
$$

In [11], Gökhan Çuvalcıoğlu introduced operator $E_{\alpha, \beta}$ that has the following IVIFPform

$E_{\alpha, \beta}(x)=\langle[\beta(\alpha \inf M+1-\alpha), \beta(\alpha \sup M+1-\alpha)],[\alpha(\beta \inf N+1-\beta), \alpha(\beta \sup N+1-\beta)]\rangle$.

All assertions, proved for the IFS- and for the IFP-cases are valid in IVIFP-case, too (see, e.g., $[5,6,7,10]$. For example, we can prove 
Theorem 5.1 (cf. [8]). The two most extended modal operators

$$
X\left(\begin{array}{llllll}
a_{1} & b_{1} & c_{1} & d_{1} & e_{1} & f_{1} \\
a_{2} & b_{2} & c_{2} & d_{2} & e_{2} & f_{2}
\end{array}\right)
$$

and

$$
\square\left(\begin{array}{llllll}
\alpha_{1} & \beta_{1} & \gamma_{1} & \delta_{1} & \varepsilon_{1} & \zeta_{1} \\
\alpha_{2} & \beta_{2} & \gamma_{1} & \delta_{2} & \varepsilon_{1} & \zeta_{1}
\end{array}\right)
$$

defined over IVIFPs and satisfying (1) - (6), respectively, are equivalent.

\section{CONCLUSION}

In the present paper, we transform the definitions of the basic relations, operations and operators to the concept of an IVIFP.

In future, we will give definitions of new operations and operators over IVIFPs.

In [4], it is shown that the IFSs are a suitable tool for evaluation of Data mining processes and objects. We plan to discuss the possibilities to use IVIFPs as a similar tool.

\section{ACKNOWLEDGMENTS}

The first two authors are thankful for the support provided by the Bulgarian National Science Fund under Grant Ref. No. DN-02-10/2016 "New Instruments for Knowledge Discovery from Data, and their Modelling" and the Program for career development of young scientists, Bulgarian Academy of Sciences, Grant number DFNP-17-139 "Investigation of different extensions of fuzzy sets and assessment of their correctness".

\section{REFERENCES}

[1] Angelova, N., M. Stoenchev. Intuitionistic fuzzy conjunctions and disjunctions from first type, Annual of "Informatics" Section, Union of Scientists in Bulgaria, Vol. 8, 2015/2016, 1-17.

[2] Atanassov, K. Intuitionistic Fuzzy Sets, Springer, Heldelberg, 1999.

[3] Atanassov, K. On Intuitionistic Fuzzy Sets Theory, Springer, Berlin, 2012.

[4] Atanassov, K. Intuitionistic fuzzy logics as tools for evaluation of Data Mining processes, Knowledge-Based Systems, Vol. 80, 2015, 122-130.

[5] Atanassov, K. Intuitionistic fuzzy modal operators of second type over interval-valued intuitionistic fuzzy sets. Part 1, Notes on Intuitionistic Fuzzy Sets, Vol. 24, 2018, No. 2, 8-17.

[6] Atanassov, K. Intuitionistic fuzzy modal operators of second type over interval-valued intuitionistic fuzzy sets. Part 2, Notes on Intuitionistic Fuzzy Sets, Vol. 24, 2018, No. 3 (in press).

[7] Atanassov, K. On the Most Extended Modal Operator of First Type over Interval-Valued Intuitionistic Fuzzy Sets. Mathematics 2018, 6, 123; doi:10.3390/math6070123

[8] Atanassov, K.On the two most extended modal types of operators defined over interval valued intuitionistic fuzzy sets. Annals of Fuzzy Mathematics and Informatics (in press).

[9] Atanassov, K., E. Szmidt, J. Kacprzyk. On intuitionistic fuzzy pairs, Notes on Intuitionistic Fuzzy Sets, Vol. 19, 2013, No. 3, 1-13

[10] Atanassov, K., Szmidt, E., Kacprzyk, J., Vassilev, P. On intuitionistic fuzzy pairs of n-th type. Issues in IFSs and GNs, 13, 2017, ISBN:978-83-61551-21-8, 136-142.

[11] Çuvalcioğlu, G., Some properties of $E_{\alpha, \beta}$ operator. Advanced Studies in Contemporary Mathematics, Vol. 14, 2007, No. 2, 305-310.

[12] Feys R. Modal Logics, Gauthier-Villars, Paris, 1965.

[13] Szmidt, E., Distances and Similarities in Intuitionistic Fuzzy Sets, Springer, Berlin, 2014.

[14] Vassilev, P., Ribagin, S., Kacprzyk, J. (2018) A remark on intuitionistic fuzzy implications. Notes on Intuitionistic Fuzzy Sets, 24 (2), 1-7, doi: 10.7546/nifs.2018.24.2.1-7 
(Krassimir Atanassov, Peter Vassilev) Dept. of Bioinformatics and Mathematical Modelling Institute of Biophysics and BiomedicAl ENGINEERING, BULGARIAN ACADEMY OF SCIENCES 105 ACAD. G. BOnchev Str., 1113 Sofia, Bulgaria,

E-mail address, Krassimir Atanassov: krat@bas .bg

E-mail address, Peter Vassilev: peter.vassilev@gmail.com

(Eulalia Szmidt, Janusz Kacprzyk) Systems Research Institute - Polish ACAdemy of Sciences,, Ul. NEWELSKa 6, 01-447 Warsaw, POLAND

E-mail address, Eulalia Szmidt: szmidt@ibspan.waw.pl

E-mail address, Janusz Kacprzyk: kacprzyk@ibspan.waw.pl 